\title{
norden
}

\section{Nordic plastic value chains}

Case WEEE (Waste Electrical and Electronic Equipment)

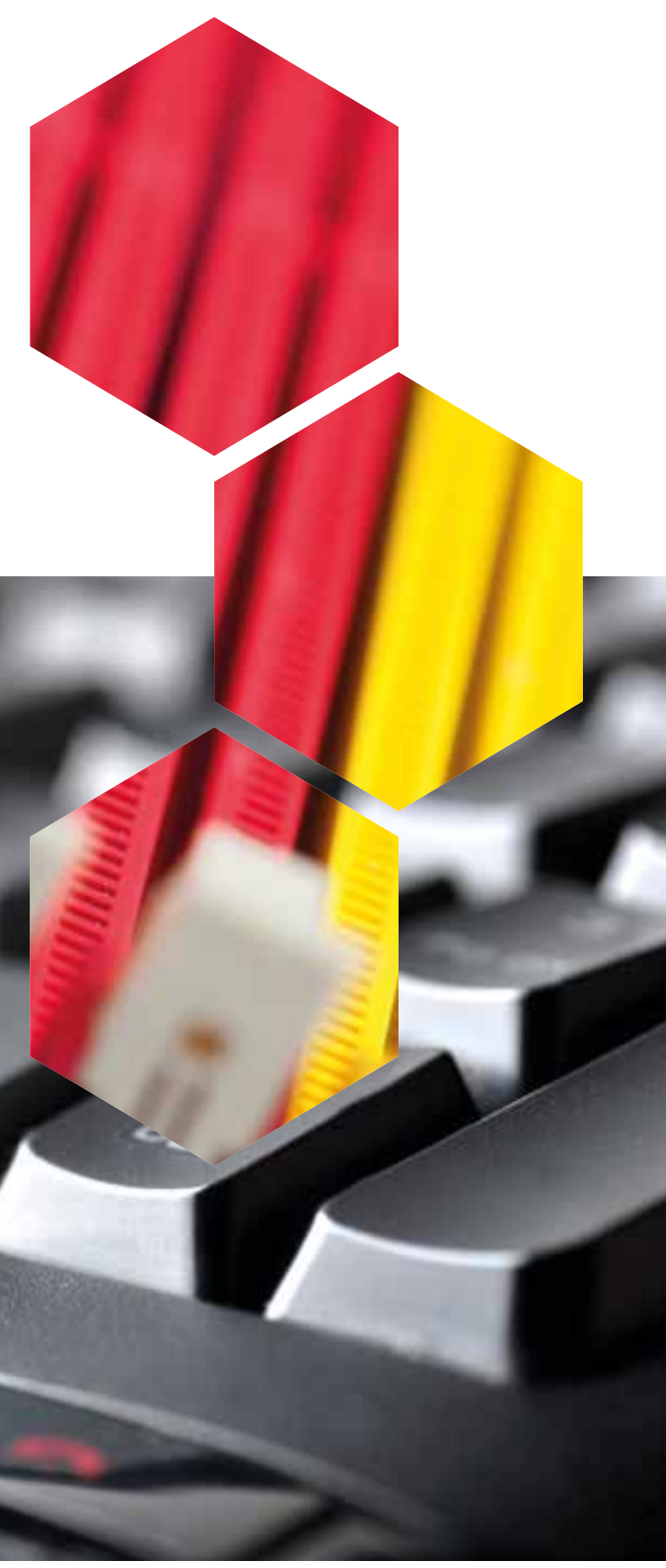

/Policy brief 


\section{Nordic plastic value chains}

Case WEEE (Waste Electrical and Electronic Equipment)

ISBN 978-92-893-3977-3 (PRINT)

ISBN 978-92-893-3978-0 (PDF)

http://dx.doi.org/10.6027/ANP2015-718

ANP 2015:718

(C) Nordic Council of Ministers 2015

Authors: John Baxter, Aina Stensgård and Hanne L Raadal, Ostfold Research, Anna Fråne, IVL Swedish Environmental Research Institute, Malin zu Castell Rüdenhausen and Margareta Wahlström, VTT Technical Research Centre of Finland, Massimo Pizzol and Søren Løkke, Aalborg University, Stefán Gíslason, Environice Layout: Gitte Wejnold

Cover photo: SignElements

Photo: SignElements

Print: Rosendahls-Schultz Grafisk

Copies: 100

Typeface: Meta LF

Paper: Munken Polar

Printed in Denmark

www.norden.org/en/publications

\section{Nordic co-operation}

Nordic co-operation is one of the world's most extensive forms of regional collaboration, involving Denmark, Finland, Iceland, Norway, Sweden, and the Faroe Islands, Greenland, and Åland.

Nordic co-operation has firm traditions in politics, the economy, and culture. It plays an important role in European and international collaboration, and aims at creating a strong Nordic community in a strong Europe.

Nordic co-operation seeks to safeguard Nordic and regional interests and principles in the global community. Common Nordic values help the region solidify its position as one of the world's most innovative and competitive.

\section{Nordic Council of Ministers}

Ved Stranden 18

DK-1061 Copenhagen K

Telefon (+45) 33960200

www.norden.org 

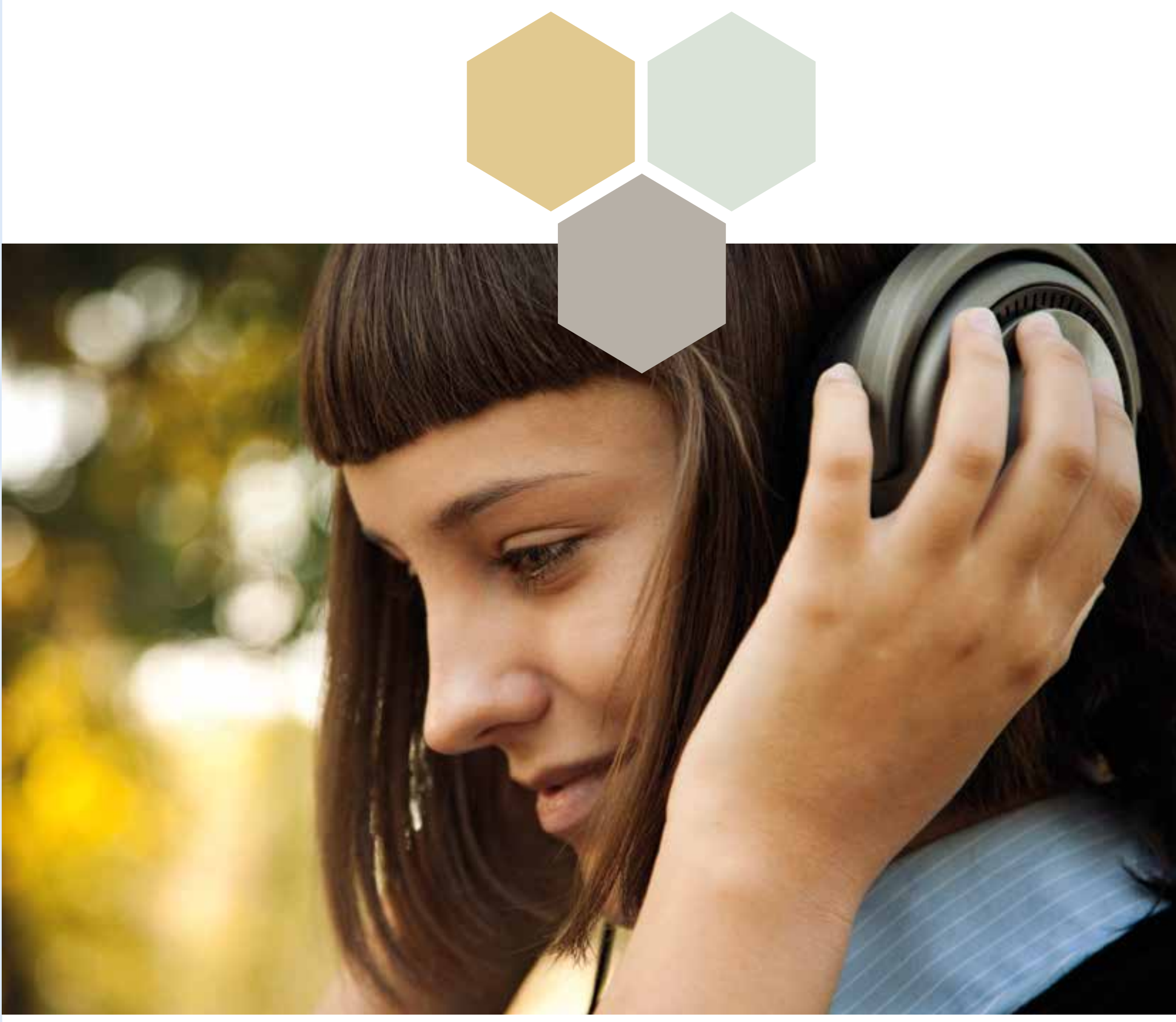


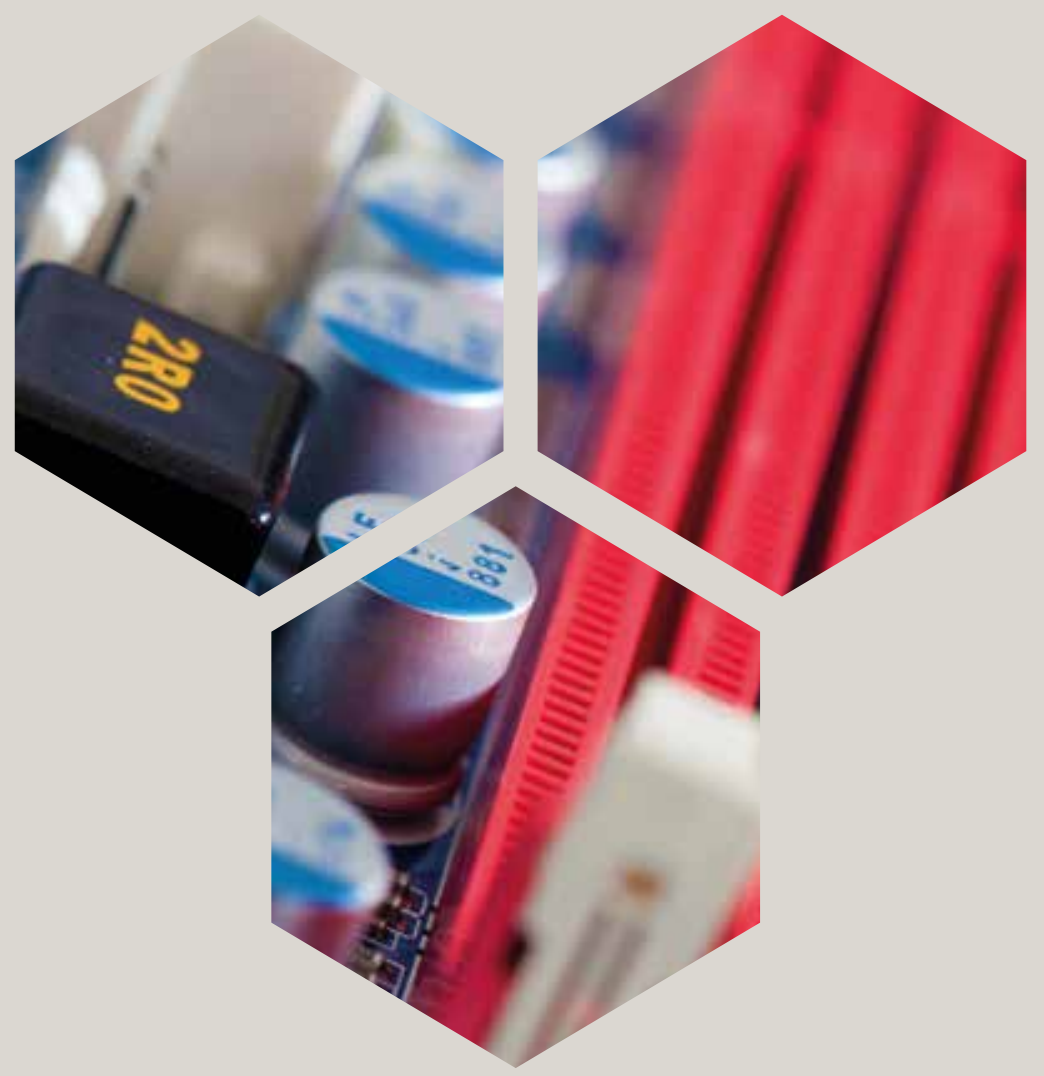




\title{
Nordic plastic value chains
}

\author{
Case WEEE (Waste Electrical and \\ Electronic Equipment)
}

Policy brief

John Baxter, Aina Stensgård and Hanne L Raadal, Ostfold Research Anna Fråne, IVL Swedish Environmental Research Institute Malin zu Castell Rüdenhausen and Margareta Wahlström , VTT Technical Research Centre of Finland Massimo Pizzol and Søren Løkke, Aalborg University Stefán Gíslason, Environice 


\section{Introduction}

\author{
Waste Electrical and Electronic \\ Equipment, WEEE, differs from \\ other types of wastes due to its \\ complex composition of various \\ materials. Valuable metals, plastics, \\ pollutants and other substances \\ are mixed together in to one single \\ product. This complexity poses some \\ challenges for both the collection \\ and recycling of WEEE. Although \\ overall WEEE material recycling rates \\ are relatively high, they are largely \\ based on the recovery of valuable \\ materials, such as metals. For more \\ challenging WEEE elements, notably \\ plastics, the recycling rates are much \\ lower.
}

After metal, plastic is the main component and makes up for about $15 \%$ of the WEEE-volume. Although recycling of plastics is considered to be an important environmental strategy, it is thought that well under $25 \%$ of WEEE plastics are recycled. Hazardous compounds in plastics present some barriers, but much of the shortcoming in recycling can be attributed to technical and economic issues.

This brief sums up the main findings from the project Nordic plastic value chains - Case WEEE (Waste Electrical and Electronic Equipment), in a policy perspective. The aim of this project is to provide an overview of the WEEE plastic waste situation in the Nordic countries, with a view to proposing improvements along the value chain. The project is one of three plastics recycling projects within the Nordic Prime Ministers' green growth initiative, The Nordic Region - leading in green growth.

The project's end result is an extensive report divided into five themes that were identified as potential areas for improvement. This policy brief summarizes the key findings for each theme, the report's recommendations and general conclusion. The themes concern: the appropriate direction of waste along the value chain; the traceability of plastics through the value chain; the technology mix for plastics recycling; design for recycling and environmental declarations; features of the Extended Producer Responsibility scheme.

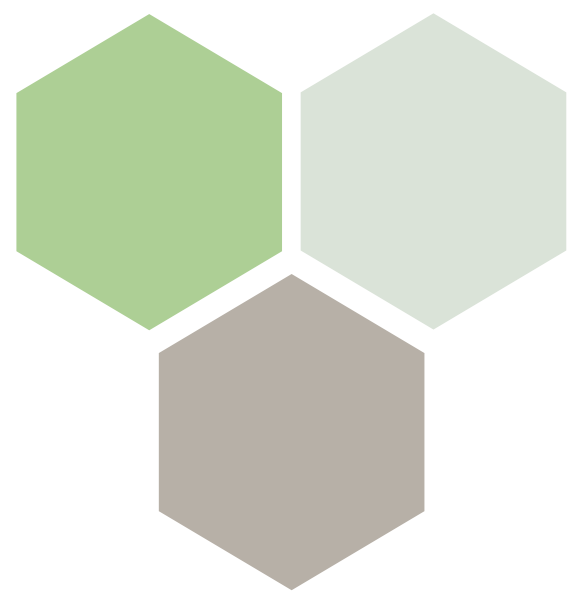




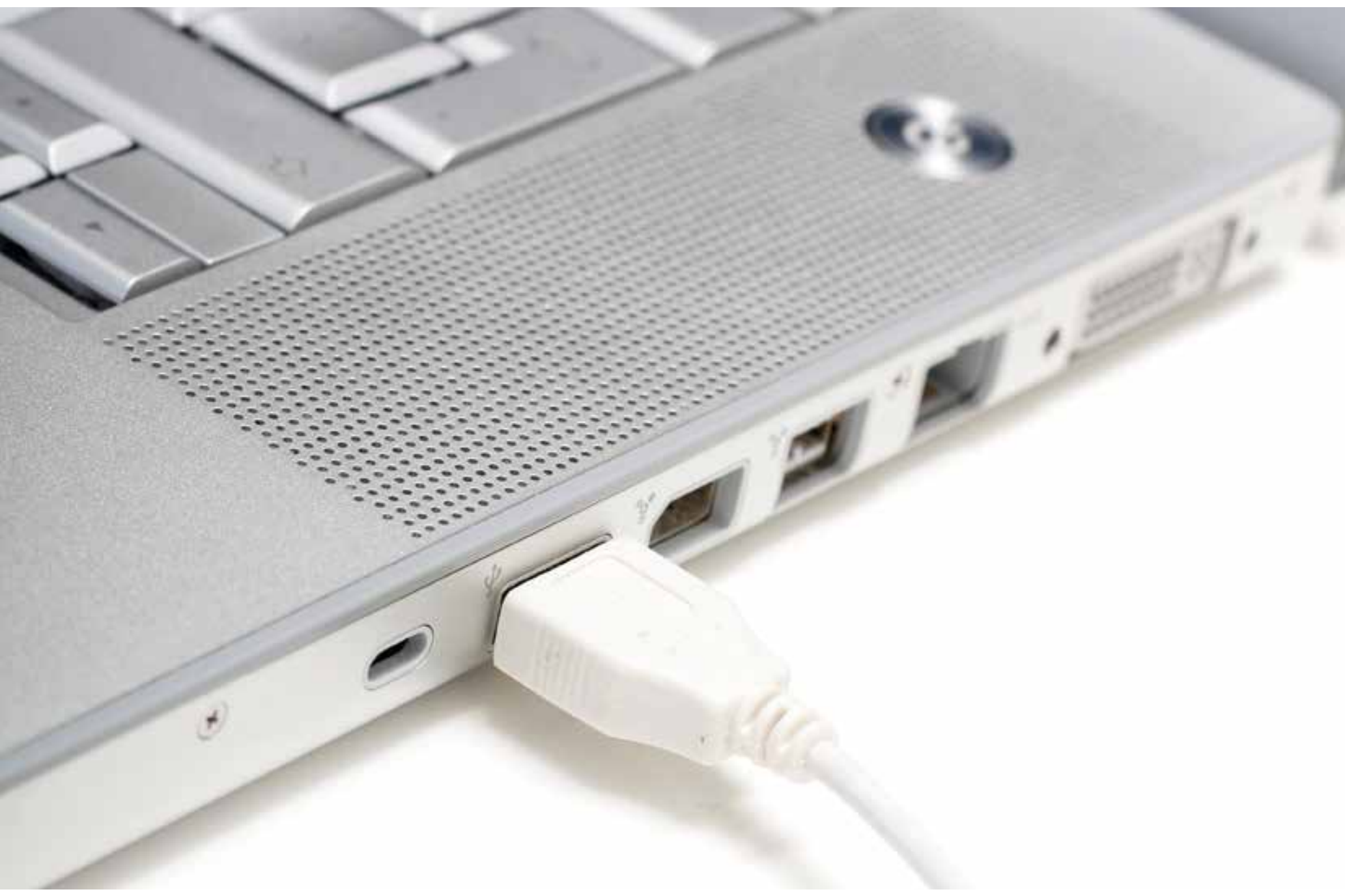


these could be as simple as specific collection and recycling targets for particular materials.

\section{Extended Producer Responsibility schemes should be structured so as to promote Design for Recycling}

Design for recycling (DFR) is not particularly encouraged by EPR schemes as they currently stand, and there are even some tangible disincentives, such as equal EPR fees for manufacturers, meaning that those engaging in DFR are effectively subsidising those who do not. The environmental advantages of design for recycling could be calculated and communicated within the marketplace. This could lead to some realisable benefits for electronics producers. However, it seems clear that EPR schemes themselves can do more to promote design for recycling. The general drive towards higher-quality treatment, perhaps coupled with regulations designed to reward good practice rather than merely establish minimum threshold standards, should in the long-term promote a culture where design for recycling is positively encouraged.

\section{Communication and partnership across and along the value chain is essential}

In the first instance, the above recommendations depend fundamentally on "unilateral" action by governments and regulatory bodies - in that improvements in practice will necessarily be driven by regulations. However, this in itself requires considerable consensus between different national authorities, and in turn with the sector as a whole. In practice, ongoing developments in all the above areas are also dependent on co-operation from actors at all parts of the value chain. Fora such as the workshops and events organised by the Nordic Council, both within the plastics recycling projects and otherwise, provide great opportunities for building the necessary networks and partnerships. More such events should be organised on a regular basis. 


\section{Key findings}

The above recommendations for improving recycling of plastics WEEE are based on the following key findings.

\section{Overall direction of waste}

One of the main challenges for WEEE plastic recycling is that portions of the waste never enters the official recycling value chain established by the Extended Producer Responsibility-scheme (EPRscheme). WEEE moves along several paths, which makes it difficult for the official system to collect the waste. In addition to the "official WEEE-stream", there are metal scrap dealers and other stakeholders that are interested in certain fractions of the WEEE - including legal and illegal actors. Furthermore, a part of the WEEE-stream ends up in the residual waste flow and some is stored in households. A common feature of all these diverted pathways is that they entail little or no plastics recycling. The net positive material value of certain WEEE fractions is attractive to actors outside the official value chain. When WEEE is diverted from the official waste stream, value is constantly being extracted from the EPR-scheme. Illegal export of WEEE is probably the biggest threat, both for the environment and for the EPR-system. Better policing of waste exports is therefore needed and would probably be the single most important factor for keeping waste in the system. WEEE is also (dubiously) shipped as used EEE. The relevant regulations for classifying and labelling used EEE and WEEE probably need careful reexamination.

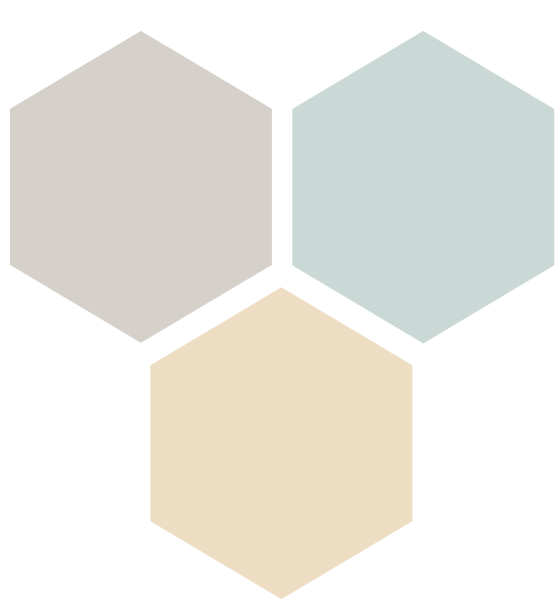

\section{Traceability}

Despite the WEEE legislation on treatment and restrictions, WEEE management suffers from lack of traceability. This means that it is difficult to link and keep track of input and output waste flows in WEEE management systems, and more in general it is difficult to keep track of input and output material flows in the EEE-WEEE supply chain. The drawback of this situation is that reporting becomes more complicated and imprecise, and that legal compliance is not guaranteed. Full traceability of WEEE management should allow increased collection rates, reduce uncontrolled flows such as illegal shipping to developing countries, and ensure proper treatment of the materials according to their plastic composition and hazardous substance content. 
Traceability of wastes through the value chain could be radically improved (at additional cost) using essentially existing infrastructure. This could have substantial positive influences on the quality of WEEE processing and treatment, and ultimately recovery levels of many materials including plastics. In part the change would imply more assiduous implementation of existing policies and protocols - for example in record-keeping, measurement or analysis.

Otherwise, this could include new technologies and innovation such as smart labelling. This will allow both existing regulatory drivers to be satisfied but also facilitate increasing quality in the treatment value chain.

\section{Technology}

Most actors in the WEEE sector indicate that technological issues are not at the forefront of considerations, with market and regulatory issues being more important. Nonetheless, technology issues remain important with particular respect to recycling of WEEE plastics, as the current technological mix does not seem entirely fit for purpose, particularly in terms of effectively separating plastics containing hazardous material (brominated flame retardants). Regulatory restrictions mean that such materials are effectively non-recoverable, and it seems that substantial quantities of otherwise "clean" plastics are lost in eliminating such material from the value chain.
Evidence suggests that WEEE treatment processes with significant low-tech elements, including manual disassembly and separation of WEEE, can currently achieve significantly better plastics recycling than highly mechanised and automated alternatives - meaning more selective and accurate separation, implying reduced loss of clean plastics with contaminated fractions. Lower-tech approaches carry relatively higher costs and may otherwise appear unattractive in a sector where technological advance seems vital. However, WEEE plastics are especially challenging to recycle and depend on high performance processes.
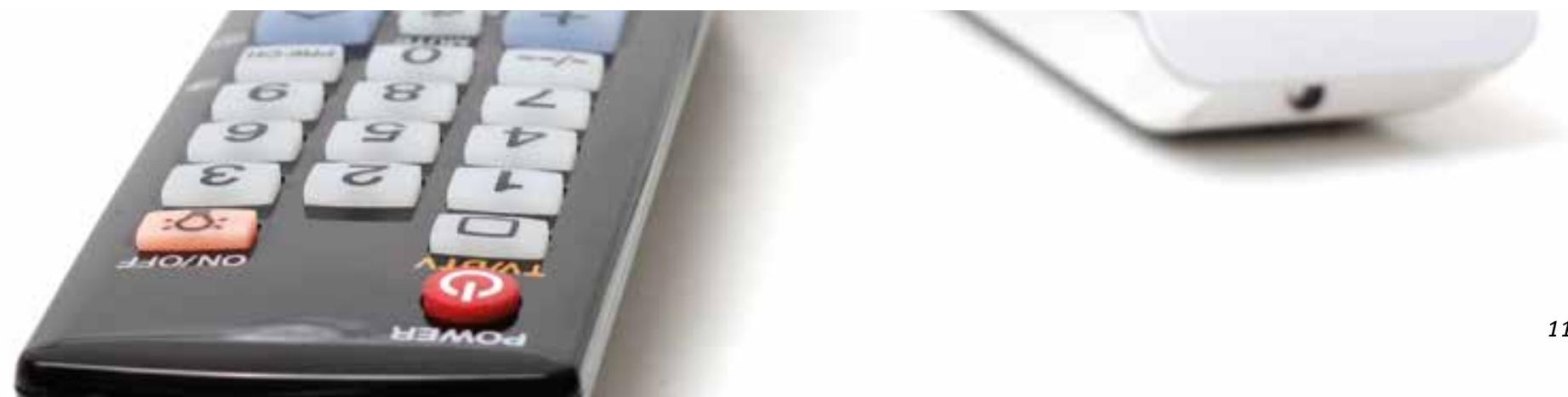
Changes in regulation and/or the funding climate could bring plastics recycling into greater focus and possibly make low-tech, costly, yet high-quality plastics recycling much more attractive than it is at present. Recyclers should be wary of losing their capability for low-tech WEEE recycling; it may be needed in the future.

\section{Environmental declarations}

There seems to be little general push towards design for recycling in consumer electronics. However, analysis shows that improved design with respect to plastics, via recycled plastics feedstock, and/ or design to ensure recyclability of products at end-of-life, yields tangible environmental advantages. This could reduce the product environmental impact by $20 \%$ or more. The savings could be even greater if other components including metals also are taken into account.

A key factor is that such environmental savings should be communicable to the market and/ or consumers through product environmental declarations, and hence offer market and economic advantages for electronics manufacturers. Some manufacturers have taken steps to explicitly introduce such practices, whilst other manufacturers have the general tenets embedded in their strategic outlook.

\section{Motivating the public is essential}

The current collection systems in the Nordic countries are highly based on source-separation of WEEE, making them dependent on the consumers' behaviour and willingness to contribute to the system. One of the challenges is that large amounts of 
WEEE are piled up in the society, making recycling impossible. Accumulated WEEE represents both a potential environmental hazard and an important resource stock. Motivating the public to hand in small WEEE is therefore a key challenge for increased recycling of WEEE plastics. Also public awareness on how, where and what to recycle is essential to achieve higher collection rates.

\section{Policy instruments to be considered}

In addition to challenges concerning the consumer's behaviour, the economic design of the EPR-scheme also poses some complications. Currently the funding of WEEE treatment through EPR schemes only reflects what is done and how it is done, i.e. it only reflects the WEEE in the scheme that is collected and recycled, while the WEEE outside the scheme is appraised.

At the same time, overall collection targets regulate the amount of material collected. These factors provide no incentive for recyclers or other actors to meet more than minimum threshold standards. Additionally, the targets being weight-based means that WEEE plastic is particularly vulnerable to being overlooked or ignored. In theory the change of collection targets to being expressed as proportions of material put on the market should drive increased collection and recycling, but the regulatory drivers look far too crude and coarse-grained to truly incentivise better practice.

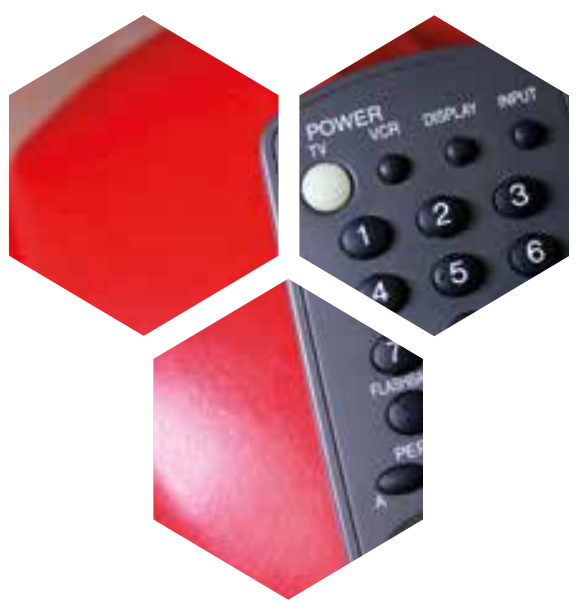




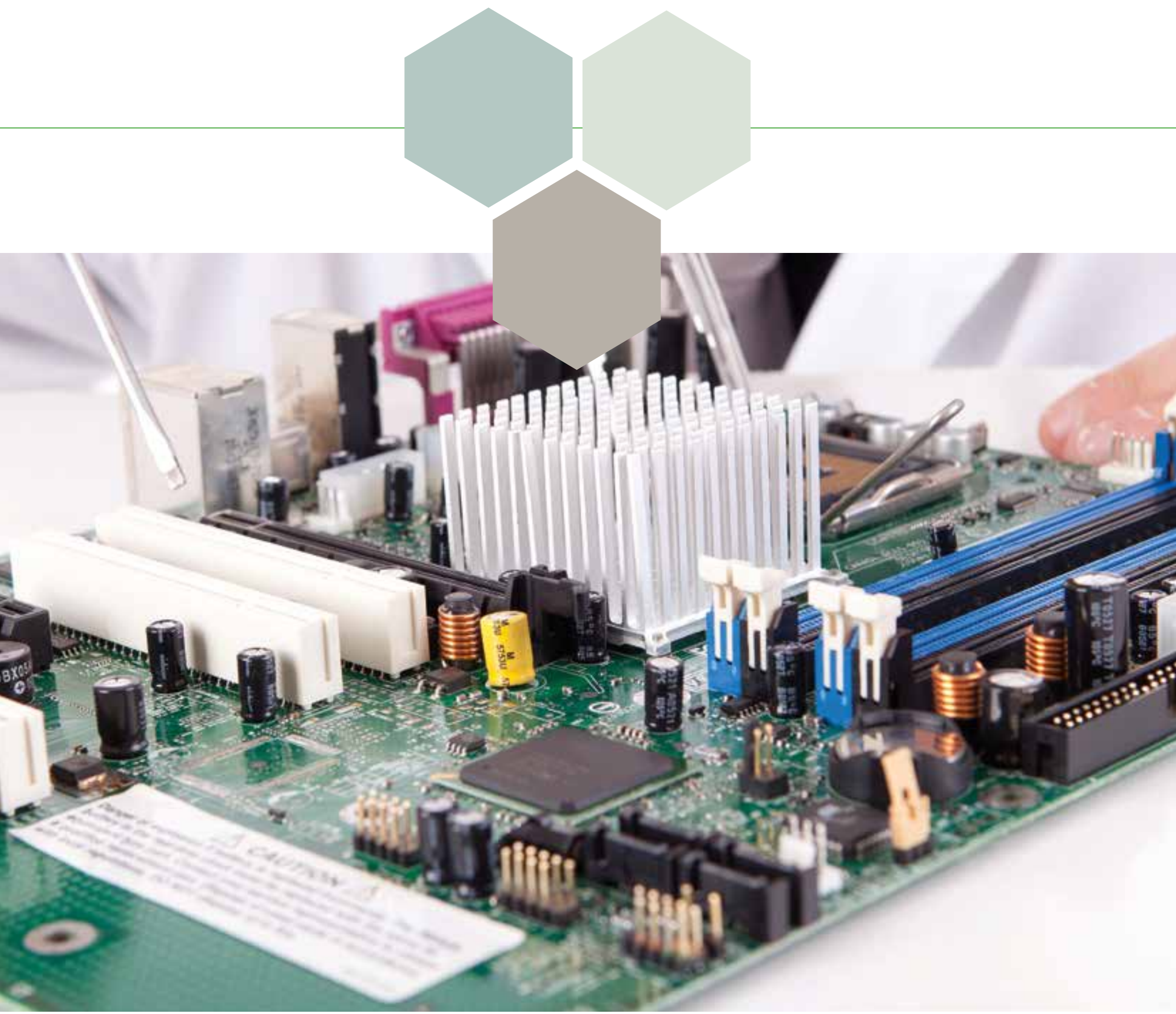




\section{Conclusion}

Several of the factors for improved recycling of WEEE plastics are concerned with WEEE more generally than with plastics specifically. This shows that WEEE plastics recycling cannot simply be improved in isolation, but has to be a result of measures and adjustments throughout the WEEE-value-chain.

One of the main challenges identified for recycling of WEEE plastics, is the need of consumer awareness. What is electronic waste, and what to do with such waste at end-of-life, is crucial to guide to the consumers in order to get these waste flows into the official WEEEsystem. Still, recyclers are somewhat dependent on the actions of other stakeholders. In particular, they may be required to bear increased costs, both for the treatment itself and for the administration, tracking and monitoring of waste through their systems. They cannot realistically be expected to do this alone; they need co-operation from many other parties, and specific financial drivers arising from policy-makers and/or producers.

In addition to changes in consumer behavior, the EPR-scheme itself is in need of some adjustments. The overall funding level of EPRschemes and hence the financial flows through such schemes are essentially arbitrary or subjectively chosen. For WEEE, tariff levels (those fees paid by producers to fulfill their extended responsibility) supposedly reflect the true net costs of waste processing and treatment. However, the extent and quality of waste treatment, and hence the cost level, is a matter of choice. European EPR schemes for WEEE are low-cost with consequent compromises on the quality of treatment. Costlier, higherquality alternatives would give rise to many substantial advantages over existing schemes, not least in the environmental perspective. Additional costs would be at least partly offset by the additional benefits of higher quality waste treatment. Substantial enhancement of WEEE plastics recycling is probably not possible without some restructuring of the cost base. 


\section{norden}

Nordic Council of Ministers

Ved Stranden 18

DK-1061 København K

www.norden.org

This policy brief on recycling of WEEE, Waste Electrical and Electronic Equipment, is part of the Nordic Prime Ministers' green growth initiative, The Nordic Region - leading in green growth. Read more about the initiative in the web magazine Green Growth the Nordic Way at www.nordicway.org or at www.norden.org/greengrowth

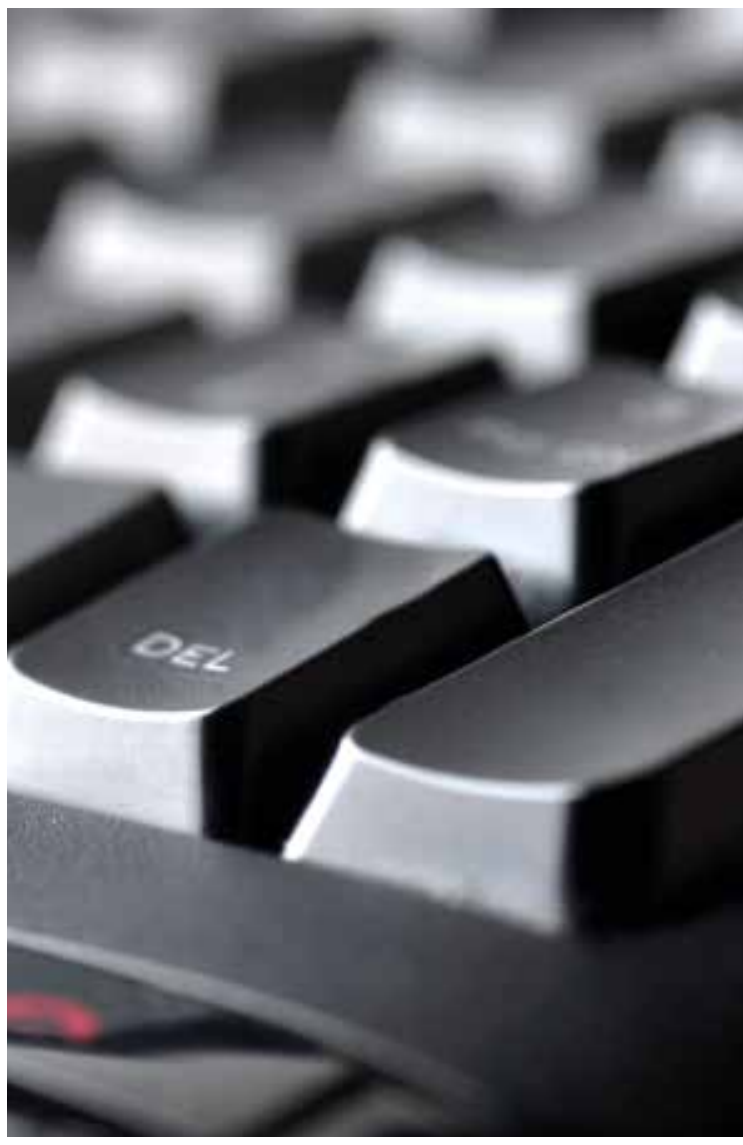

